University of Wollongong

Research Online

Faculty of Engineering - Papers (Archive)

Faculty of Engineering and Information

Sciences

1999

\title{
Central-cell corrections for Si and S in GaAs in a strong magnetic field
}

R. J. Heron

University of Wollongong

R. A. Lewis

University of Wollongong, roger@uow.edu.au

Philip E. Simmonds

University of Wollongong, simmonds@uow.edu.au

R. P. Starrett

University of New South Wales

A. V. Skougarevsky

University of New South Wales

See next page for additional authors

Follow this and additional works at: https://ro.uow.edu.au/engpapers

Part of the Engineering Commons

https://ro.uow.edu.au/engpapers/458

\section{Recommended Citation}

Heron, R. J.; Lewis, R. A.; Simmonds, Philip E.; Starrett, R. P.; Skougarevsky, A. V.; Clark, R G.; and Stanley, C. R.: Central-cell corrections for Si and S in GaAs in a strong magnetic field 1999.

https://ro.uow.edu.au/engpapers/458

Research Online is the open access institutional repository for the University of Wollongong. For further information contact the UOW Library: research-pubs@uow.edu.au 
Authors

R. J. Heron, R. A. Lewis, Philip E. Simmonds, R. P. Starrett, A. V. Skougarevsky, R G. Clark, and C. R. Stanley 


\title{
Central-cell corrections for $\mathrm{Si}$ and $\mathrm{S}$ in $\mathrm{GaAs}$ in a strong magnetic field
}

\author{
R. J. Heron, ${ }^{\text {a) }}$ R. A. Lewis, and P. E. Simmonds \\ Department of Physics, University of Wollongong, Wollongong, New South Wales 2522, Australia \\ R. P. Starrett, A. V. Skougarevsky, and R. G. Clark \\ School of Physics, University of New South Wales, Kensington, New South Wales 2052, Australia \\ C. R. Stanley \\ Department of Electronics and Electrical Engineering, University of Glasgow, Glasgow G12 8TL, \\ United Kingdom
}

(Received 15 June 1998; accepted for publication 12 September 1998)

\begin{abstract}
The central-cell correction has been determined experimentally for the two donor impurities $\mathrm{S}$ and $\mathrm{Si}$ in GaAs. Data have been obtained for magnetic fields to $39 \mathrm{~T}$, corresponding to $\gamma \approx 6$. The observed behavior is in good agreement with theory. The analysis permits accurate evaluation of zero-field central-cell corrections, yielding 0.110 and $0.059 \mathrm{meV}$ for $\mathrm{S}$ and $\mathrm{Si}$, respectively. (C) 1999 American Institute of Physics. [S0021-8979(98)02724-8]
\end{abstract}

\section{INTRODUCTION}

The electrical and optical properties of GaAs that lead to its technological importance depend in a large part on the impurities it contains, either deliberately or unintentionally introduced. Shallow donors, especially $\mathrm{Si}$, are of continuing interest. $^{1-3}$ The usual framework for understanding the energy states of shallow donors is the effective-mass approximation which leads to a hydrogen-like energy spectrum. This article examines as a function of magnetic field one correction that needs to be applied to the hydrogenic model to fit the observed spectra, that due to the chemical identity of the donor.

In applying the hydrogenic model to $n$-GaAs, it is necessary first to scale the hydrogen energy levels by the appropriate materials parameters. The dielectric constant of GaAs, $\epsilon=12.56$, and the effective mass of GaAs conduction electrons, $m^{*}=0.0665 m_{e}$, yield an effective Rydberg of $\mathrm{Ry}^{*}=5.72 \mathrm{meV}$. Likewise, the dimensionless magnetic field $\gamma \equiv \frac{1}{2} \hbar \omega_{c} / \mathrm{Ry} *$, where $\omega_{c}=e B / m^{*}$, has a value of unity in $n$-GaAs for magnetic field $B=6.57 \mathrm{~T}$; this defines an "intermediate" magnetic field. Second, additional factors, not applicable in the case of hydrogen, need to be introduced. These include band nonparabolicity, ${ }^{4,5}$ band anisotropy, ${ }^{4,6}$ and polaron effects. ${ }^{7}$ These effects become more pronounced as the energy of the states increases.

An unavoidable correction to the simple hydrogenic model is the central-cell correction. This arises due to differing potentials at the impurity site. This perturbation is present even in the absence of a magnetic field. Zero-field central-cell corrections have been extensively studied with a view to identifying the different chemical species involved. ${ }^{8-14}$ Compared to this large amount of work on the zero-field case, the magnetic-field dependence of the centralcell correction has been subject to little study. An early report gave the difference in chemical shift for two unidentified donors. ${ }^{15}$ Other works have used this data ${ }^{11}$ or simply a

\footnotetext{
${ }^{a)}$ Electronic mail: rjh@newt.phys.unsw.edu.au
}

$B^{2}$ dependence ${ }^{16}$ to model the effect. These reports are limited to fields below $7 \mathrm{~T}$. Even less data are available at high magnetic fields. While measurements on $n$-GaAs have been reported to $150 \mathrm{~T}$, the analysis has revealed only nonparabolicity and anisotropy, and not central-cell effects. ${ }^{17}$ Unidentified donors in InSb have been studied to $20 \mathrm{~T}(\gamma \approx 130){ }^{18}$ In contrast to that study, the present article deals with the behavior of two known donors in the technologically important material GaAs. It might be noted that, apart from its inherent interest, the magnetic-field dependence of the central-cell correction permits a means for a more accurate evaluation of the zero-field effect, in that a number of data, taken at various fields, can be fitted by the theory to yield the zero-field result.

Polaron effects, which become significant at energies approaching those of the optical phonons of the host lattice, and band nonparabolicity, the effects of which increase with energy, confuse the measurement of central-cell corrections in higher-energy transitions, e.g., $1 s \rightarrow 2 p_{0}$ and $1 s \rightarrow 2 p_{+1}$. To minimize these effects the hydrogenic transition of lowest energy, $1 s \rightarrow 2 p_{-1}$, is studied here. Values for central-cell corrections derived from this transition have not been reported previously.

\section{THEORY}

The energy levels of the hydrogen atom in an arbitrary magnetic field are of inherent theoretical interest as well as in condensed matter physics. The area has been reviewed by Garstang. ${ }^{19}$ Very accurate calculations have been reported by Rösner et al. ${ }^{20}$ The results of Makado and McGill ${ }^{21}$ include energies for a large number of states at a large number of magnetic fields. The uncertainty in these calculated results is much less than that in the experimental data.

The energy levels of chemically distinct donors differ slightly due to slightly different potentials present at the impurity site. The observed chemical shift in transition energies 
is attributed entirely to the even-parity ground state, the oddparity final states having no chemical shift since their wave functions have zero amplitude at the impurity site. The central-cell correction takes into account the probability of finding the electron in a small volume near the impurity center. With increasing magnetic field the electron is squeezed into a smaller volume, increasing the effect. Fetterman et al. ${ }^{15}$ have pointed out that, using lowest order perturbation theory, the central-cell correction for a donor of species $j$ may be written as

$$
\Delta E_{j}=\kappa_{j}|\Phi(0)|^{2} .
$$

Here $\kappa_{j}$ is a constant which depends on the particular donor species and $\Phi(\mathbf{r})$ is the appropriate envelope function appearing in the effective-mass wave function of the hydrogenic ground state. According to Eq. (1), all donor species show the same relative change in $\Delta E_{j}$ as magnetic field, and so $|\Phi(0)|^{2}$, varies. Values of $|\Phi(0)|^{2}$ have been calculated and tabulated for a wide range of magnetic fields by Cabib, Fabri, and Fiorio. ${ }^{22}$

\section{EXPERIMENT}

The high magnetic fields employed in this investigation were generated at the Australian National Pulsed Magnet Laboratory, a full description of which is given elsewhere. ${ }^{23}$ In brief, a $32 \mu \mathrm{F}$ capacitor bank, charged to as much as $7 \mathrm{kV}$, is discharged through a liquid nitrogen-cooled copper-silver coil. The magnetic field rises to its peak in $\approx 10 \mathrm{~ms}$. Fields in excess of $60 \mathrm{~T}$ may be produced.

The far-infrared source is a molecular-gas cavity pumped by a $\mathrm{CO}_{2}$ laser. $^{24}$ The radiation is conducted via light pipes and a top-loading probe ${ }^{25}$ to the magnet bore, where the sample is located in a ${ }^{3} \mathrm{He}$ space. Sample temperatures below $400 \mathrm{mK}$ may be maintained in this cryostat during laser irradiation. The sample mount incorporates a pick-up coil to monitor the magnetic field rise and fall and a ruthenium-oxide thermometer. Further details of the farinfrared magnetospectroscopy methods are given elsewhere. ${ }^{26}$

The high-purity sample (B54) used in this investigation is a $15 \mu \mathrm{m}$ layer grown on semi-insulating (100) GaAs by molecular-beam epitaxy, exhibiting peak mobility of $>4$ $\times 10^{5} \mathrm{~cm}^{2} \mathrm{~V}^{-1} \mathrm{~s}^{-1}$ in the temperature range $28-42 \mathrm{~K}$. Details of the growth conditions and electrical characterization of the sample are given elsewhere. ${ }^{27}$ Electrical contacts to the sample were made either by pressing indium dots onto the sample surface and annealing in a reducing atmosphere or by vacuum deposition of a $\mathrm{Au} / \mathrm{Ge} / \mathrm{Ni}$ alloy. Both types of contact gave equally satisfactory photoconductivity data.

\section{RESULTS AND DISCUSSION}

The low-temperature far-infrared photoconductivity spectrum of $n$-GaAs is very rich in detail. The transitions of chief interest, $1 s \rightarrow 2 p_{-1}$, track through features of several different origins as the photon energy increases-cyclotron resonance, $\mathrm{D}^{-}$transitions, ${ }^{28}$ and transitions from the $1 \mathrm{~s}$ ground state to metastable excited states. ${ }^{29}$

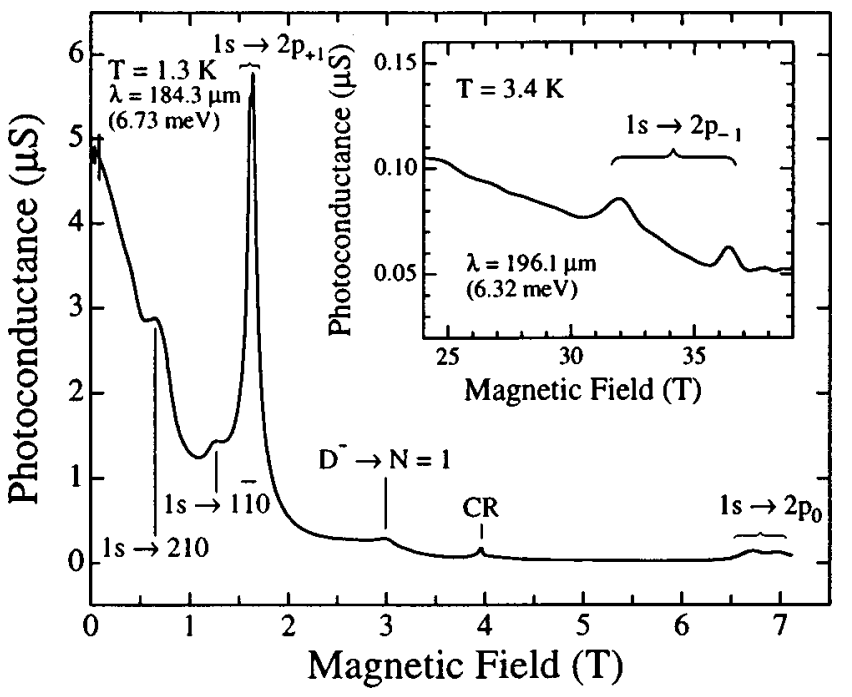

FIG. 1. Detail of photoconductivity spectra for $n$-GaAs. The main figure shows transitions associated with cyclotron resonance, $\mathrm{D}^{-}$, hydrogenic bound states $\left(1 s \rightarrow 2 p_{+1}, 1 s \rightarrow 2 p_{0}\right)$, and metastable states $[1 s \rightarrow(210)$, $1 s \rightarrow(1 \overline{10})]$. The inset shows $1 s \rightarrow 2 p_{-1}$ transitions. The ordinate has been offset. The two peaks seen on the $\left(1 s \rightarrow 2 p_{ \pm 1}, 2 p_{0}\right)$ transitions arise from chemically distinct donors, identified (Ref. 27) as S and Si.

In Fig. 1 spectra taken at excitation wavelengths of $184.3 \mu \mathrm{m}(6.73 \mathrm{meV})$ and $196.1 \mu \mathrm{m}(6.32 \mathrm{meV})$ are presented. The main features are transitions from the hydrogenlike $1 s$ ground state. Transitions from the $1 s$ to the $2 p_{+1}$, $2 p_{0}$, and $2 p_{-1}$ states are seen at about 1.6, 6.5-7, and 31-37 T, respectively, in Fig. 1. While the wave vector of the far-infrared radiation is nominally parallel to the magnetic field in our apparatus, reflection in the light pipe ensures that some component of the electric-field vector of the radiation falling on the sample is parallel to the magnetic field, allowing the observation of transitions, in particular $1 s \rightarrow 2 p_{0}$, which are strictly forbidden in the Faraday configuration. Cyclotron resonance is observed at about $4 \mathrm{~T}$ and $\mathrm{D}^{-} \rightarrow N=1$ at about $3 \mathrm{~T}$. Transitions from the $1 s$ to ( $\left.1 \overline{1} 0\right)$ and (210) metastable states may be seen at about 1.4 and 0.6 $\mathrm{T}$, respectively. At the shortest laser wavelength used, 70.5 $\mu \mathrm{m}(17.58 \mathrm{meV})$, transitions to states with index as high as (710) are observed.

The transitions from the $1 s$ to the $2 p_{+1}, 2 p_{0}$, and $2 p_{-1}$ states all show a splitting, as may be seen in Fig. 1. The splitting is due to the presence of two chemically distinct donors, identified as $\mathrm{S}$ and $\mathrm{Si}^{27}$ in the sample. The $1 s$ $\rightarrow 2 p_{-1}$ transition will be analyzed in detail since, occurring at lower energy than the $2 p_{0}$ and $2 p_{+1}$ transitions, it is less affected by nonparabolicity and polaron effects. Furthermore, it remains in the far-infrared to higher magnetic fields.

The difference in the experimental energy of the $1 \mathrm{~s}$ $\rightarrow 2 p_{-1}$ transition and the generic energies obtained from the hydrogen calculation is shown in Fig. 2. The theoretical value of the hydrogenic energy is obtained by interpolating data between the two fields from the given mesh of calculated values ${ }^{21}$ that bracket the experimental magnetic field. The interpolation is done separately for each chemical species. 


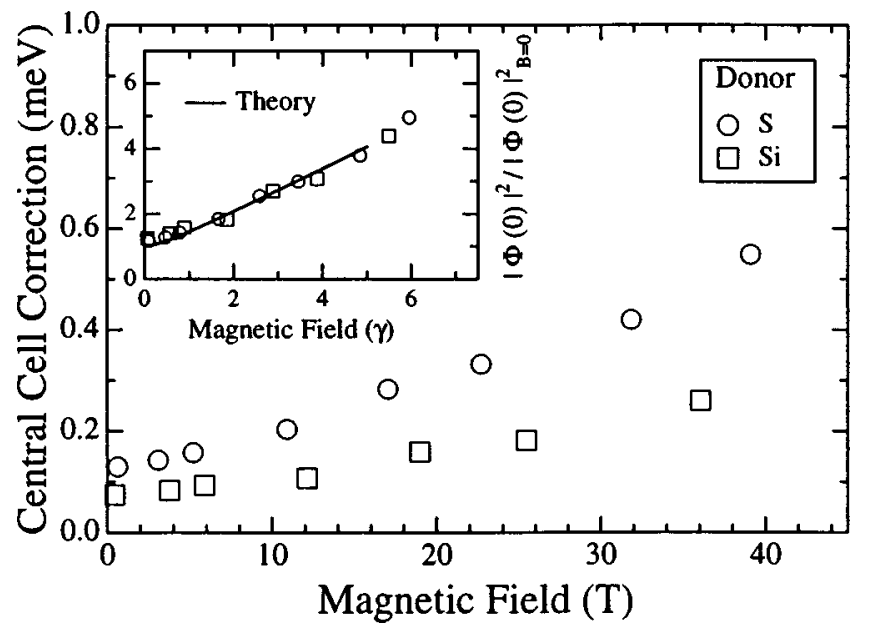

FIG. 2. Difference between experimental transition energies and energies obtained by scaling the transition energies for the hydrogen atom. Inset: Comparison of experimental data with theory Fetterman et al. (Ref. 15) using values of $|\Phi(0)|^{2} /|\Phi(0)|_{B=0}^{2}$ calculated by Cabib, Fabri, and Fiorio (Ref. 22).

It may be seen from Fig. 2 that, although each impurity has a different magnitude of chemical shift, both behave in a similar fashion as the magnetic field increases. The chemical effect increases with magnetic field due to the increasing magnetic confinement of the $1 s$ ground state.

There is little detailed experimental data reported on the field dependence of the chemical shift. While Michels et $a l .{ }^{30}$ show line positions to $15 \mathrm{~T}$, they give no values for chemical shift. Fetterman et al. ${ }^{15}$ give data for the difference in chemical shift for two unidentified donors, one of which is thought to have a negative central-cell correction. This data cannot be directly compared with the present results. The central-cell correction given by van Klarenbosch et al. ${ }^{14}$ for GaAs:Si of $0.08 \mathrm{meV}$ at zero field and $0.13 \mathrm{meV}$ at $6 \mathrm{~T}$ may be compared with the present values of 0.06 and $0.09 \mathrm{meV}$, respectively.

The inset to Fig. 2 shows the variation in central-cell correction with magnetic field according to the theoretical expression of Eq. (1) where the values of $|\Phi(0)|^{2}$ employed are those calculated by Cabib, Fabri, and Fiorio ${ }^{22}$ ratioed with the zero-field value of $|\Phi(0)|_{B=0}^{2}$. These authors give data at 16 further fields up to $\gamma=5$. These are plotted and joined to give the solid line shown.

The chemical shifts determined here by experiment are now compared directly with Eq. (1). This is accomplished by dividing the experimental fields and energies by appropriate scaling factors. The experimental magnetic fields are divided by $6.57 \mathrm{~T}$ to convert to units of $\gamma$. The experimental chemical shifts are divided by the central-cell correction at zero field. Rather than determine this quantity from a single, lowfield measurement, the experimental data over a wide range of fields are employed. For each donor, the experimental chemical shifts are least-squares fitted to the theory values (interpolated to the experimental magnetic fields) with the zero-field central-cell correction being the fitting parameter. The zero-field central-cell corrections determined in this way are $0.110 \mathrm{meV}$ for $\mathrm{S}$ and $0.059 \mathrm{meV}$ for $\mathrm{Si}$. The difference in central-cell correction between the two species presumably reflects the difference in atomic volume, although we have not made and do not know of a detailed calculation of the size of the effect. Thus the magnetic-field dependence of the central-cell correction permits a reliable evaluation of the zero-field correction since a number of data, taken at various fields, are fitted to yield the zero-field result. Moreover, the shifts at high field are greater than the small zero-field correction, again contributing to a more reliable estimate of the latter quantity than might be made in the absence of magnetic field.

It may be seen from the inset of Fig. 2 that the field dependence of the central-cell correction as expressed in Eq. (1) following Fetterman et al. ${ }^{15}$ is in good agreement with the present experimental data.

\section{CONCLUSION}

Experimentally determined chemical shifts deduced from the $1 s \rightarrow 2 p_{-1}$ transition for two donors in GaAs have been given over the range $0<\gamma<6$.

\section{ACKNOWLEDGMENTS}

The authors thank S. Butcher, and G. Facer and B. Kane for furnishing the electrical contacts on the sample, and P. Fisher for commenting on the manuscript. This work is supported in part by the Australian Research Council, the University of Wollongong, and the University of New South Wales.

${ }^{1}$ M. J. Ashwin, R. C. Newman, and K. Muraki, J. Appl. Phys. 82, 137 (1997)

${ }^{2}$ H. W. Kunert and D. J. Brink, J. Appl. Phys. 81, 6948 (1997).

${ }^{3}$ R. Apiwatwaja, R. Gwilliam, R. Wilson, and B. J. Sealy, J. Appl. Phys. 81, 1131 (1997).

${ }^{4}$ H. Sigg, J. A. A. J. Perenboom, P. Pfeffer, and W. Zawadzki, Solid State Commun. 61, 685 (1987).

${ }^{5}$ T. Ruf, and M. Cardona, Phys. Rev. B 41, 10747 (1990).

${ }^{6}$ M. A. Hopkins, R. J. Nicholas, P. Pfeffer, W. Zawadzki, D. Gauthier, J. C. Portal, and M. A. DiForte-Poisson, Semicond. Sci. Technol. 2, 568 (1987).

${ }^{7}$ A. J. van der Sluijs, K. K. Geerinck, T. O. Klaassen, W. Th. Wenckebach, and C. T. Foxon, J. Appl. Phys. 75, 3698 (1994).

${ }^{8}$ R. A. Cooke, R. A. Hoult, R. F. Kirkman, and R. A. Stradling, J. Phys. D 11, 945 (1978).

${ }^{9}$ C. J. Armistead, P. Knowles, S. P. Najda, and R. A. Stradling, J. Phys. C 17, 6415 (1984).

${ }^{10}$ G. E. Stillman, T. S. Low, and B. Lee, Solid State Commun. 53, 1041 (1985).

${ }^{11}$ A. van Klarenbosch, J. Burghoorn, T. O. Klaassen, and W. Th. Wenckebach, in Shallow Impurities in Semiconductors 1988, edited by B. Monemar, IOP Conference Proceedings No. 95 (Institute of Physics, London, 1989), p. 277.

${ }^{12}$ R. T. Grimes, M. B. Stanaway, J. M. Chamberlain, M. Henini, and O. H. Hughes, Semicond. Sci. Technol. 4, 548 (1989).

${ }^{13}$ S. P. Najda, S. Holmes, R. A. Stradling, and F. Kuchar, Semicond. Sci. Technol. 4, 791 (1989).

${ }^{14}$ A. van Klarenbosch, T. O. Klaassen, W. Th. Wenckebach, and C. T. Foxon, J. Appl. Phys. 67, 6323 (1990).

${ }^{15}$ H. R. Fetterman, D. M. Larsen, G. E. Stillman, P. E. Tannenwald, and J. Waldman, Phys. Rev. Lett. 26, 975 (1971).

${ }^{16}$ P. W. Barmby, J. L. Dunn, C. A. Bates, and T. O. Klaassen, Phys. Rev. B 54, 8566 (1996).

${ }^{17}$ S. P. Najda, S. Takeyama, N. Miura, P. Pfeffer, and W. Zawadzki, Phys. Rev. B 40, 6189 (1989).

${ }^{18}$ L. C. Brunel, S. Huant, M. Baj, and W. Trzeciakowski, Phys. Rev. B 33, 6863 (1986). 
${ }^{19}$ R. H. Garstang, Rep. Prog. Phys. 40, 105 (1977).

${ }^{20}$ W. Rösner, G. Wunner, H. Herold, and H. Ruder, J. Phys. B 17, 29 (1984).

${ }^{21}$ P. C. Makado, and N. C. McGill, J. Phys. C 19, 873 (1986),

${ }^{22}$ D. Cabib, E. Fabri, and G. Fiorio, Solid State Commun. 9, 1517 (1971).

${ }^{23}$ R. G. Clark et al., Physica B 201, 565 (1994).

${ }^{24}$ Edinburgh Instruments, Riccarton, Currie, Edinburgh EH14 4AP, UK.

${ }^{25}$ Oxford Instruments, Old Station Way, Eynsham, Witney, Oxon OX8 1TL, UK.

${ }^{26}$ R. A. Lewis, R. J. Heron, R. G. Clark, R. P. Starrett, and A. V. Skou- garevsky, in High Magnetic Fields in Semiconductor Physics II, edited by G. Landwehr and W. Ossau (World Scientific, Singapore, 1997), p. 1017.

${ }^{27}$ C. R. Stanley, M. C. Holland, A. H. Kean, M. B. Stanaway, R. T. Grimes, and J. M. Chamberlain, Appl. Phys. Lett. 58, 478 (1991).

${ }^{28}$ S. P. Najda, C. J. Armistead, C. Trager, and R. A. Stradling, Semicond. Sci. Technol. 4, 439 (1989).

${ }^{29}$ Z. Chen, Z. Chen, P. L. Liu, G. L. Shi, C. M. Hu, X. H. Shi, and S. C. Shen, J. Appl. Phys. 81, 6183 (1997).

${ }^{30}$ J. G. Michels, R. J. Warburton, R. J. Nicholas, and C. R. Stanley, Semicond. Sci. Technol. 9, 198 (1994). 